\title{
Gerontology
}

\section{Biology Explaining Tooth Repair and Regeneration: A Mini-Review}

\author{
Anamaria Balic \\ Institute of Biotechnology, University of Helsinki, Helsinki, Finland
}

\author{
Keywords \\ Tooth development $\cdot$ Aging $\cdot$ Tooth stem cells $\cdot$ Tooth \\ regeneration $\cdot$ Tooth repair
}

\begin{abstract}
The tooth is an intricate composition of precisely patterned, mineralized matrices and soft tissues. Mineralized tissues include enamel (produced by the epithelial cells called ameloblasts), dentin and cementum (produced by mesenchymal cells called odontoblasts and cementoblasts, respectively), and soft tissues, which include the dental pulp and the periodontal ligament along with the invading nerves and blood vessels. It was perceived for a very long time that teeth primarily serve an esthetical function. In recent years, however, the role of healthy teeth, as well as the impact of oral health on general well-being, became more evident. Tooth loss, caused by tooth decay, congenital malformations (tooth agenesis), trauma, periodontal diseases, or agerelated changes, is usually replaced by artificial materials which lack many of the important biological characteristics of the natural tooth. Human teeth have very low to almost absent regeneration potential, due to early loss of cell populations with regenerative capacity, namely stem cells. Significant effort has been made in recent decades to identify and characterize tooth stem cells, and to unravel the developmental programs which these cells follow in order to generate a tooth.

(c) 2018 S. Karger AG, Basel
\end{abstract}

\section{KARGER}

(c) 2018 S. Karger AG, Basel

E-Mail karger@karger.com

www.karger.com/ger

\section{Introduction}

Teeth are ectodermal organs generated by most vertebrates, and they are produced in various sizes, shapes, and numbers. With the exception of mammals, the majority of vertebrates replace their teeth continuously (polyphyodonts). Mammals, on the other hand, have restricted tooth regeneration capacity and they replace their teeth only once (diphyodonts) or not at all (monophyodonts). What restricts the arrest of tooth replacement to only one round in most mammals is not known, and the purpose or benefit of having such restriction in terms of evolution and development is not fully understood. It should be noted that most monophyodonts have continuously growing teeth, in which mineralized matrices are unceasingly produced to compensate for tooth wear.

Humans are diphyodonts which develop two dentitions during life. First is the primary dentition (also referred to as deciduous teeth), which is initiated around the 6th week of gestation. Deciduous teeth are replaced by permanent teeth, whose development is initiated between the 10th and 13th week of gestation [1]. Detailed histological analyses of human dentition have implied that humans could potentially generate a third dentition, but this potential has been inhibited by an unknown molecular mechanism. Individual teeth within the primary and secondary dentitions do not form simultaneously, 


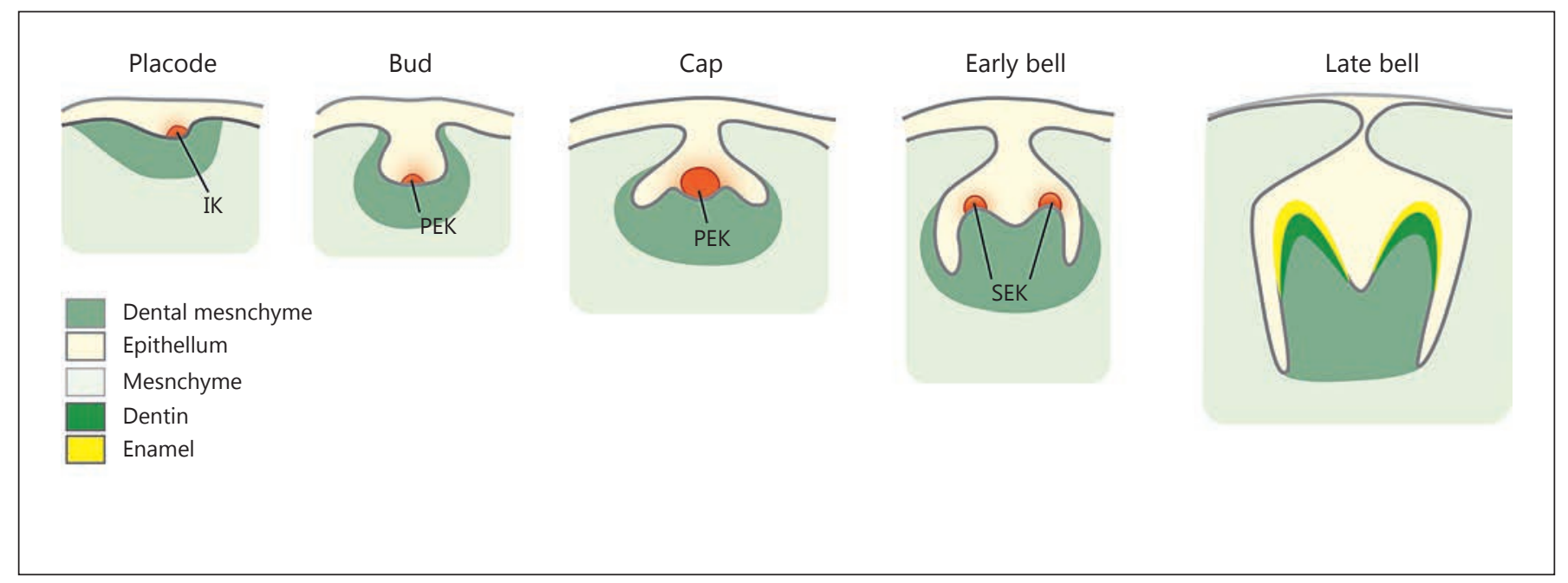

Fig. 1. Schematic presentation of tooth development. Teeth are initiated from dental lamina, a thickening of the epithelium in the upper and lower jaw. Individual tooth development is identified by the formation of a placode, from which tooth epithelium transits through bud, cap, and bell shapes which identify the distinct stages of tooth development. The morphogenesis and shape changes of the tooth epithelium are driven by signaling centers.

but in a complex temporal and spatial pattern over a span of several years which in most humans is completed in early adolescence with the development of the third molar tooth [1].

Like other ectodermal organs, teeth develop through tissue interactions mediated by the conserved signaling pathways, such as FGF, BMP, Hedhehog, Wnt, and EDA signaling pathways [2]. They also represent a valuable model to study not only development, but also gene patterning, signaling interactions, and evolution of ectodermal organs. In the past decades various signaling cascades and gene patterning schemes were resolved using the developing tooth as a model. In addition, both tooth replacement and continuous tooth growth require competent stem cells, which became the research focus in the past years. Research published in recent years has improved our understanding of tooth development and morphology, as well as the regulatory networks which govern this process. However, many questions remain unanswered, in particular those which relate to the limited reparative and regenerative potential of these intricate structures. The following text summarizes the biology behind the poor repair potential and the inability of humans to generate teeth de novo.
Three signaling centers arise during tooth development: initiation knot (IK) which forms in the placode and is accompanied by condensation of the neural crest-derived mesenchymal cells, primary enamel knot (PEK) which appears at the tip of the bud and regulates folding morphogenesis into the cap stage, and secondary enamel knots (SEK) which appear in the tips of future cusps and fine-tune the crown morphogenesis.

\section{Tooth Development in vivo}

Tooth development (Fig. 1) is governed by the reciprocal epithelial-mesenchymal interactions which occur in a step-wise process in which each stage is identified by specific morphological and cellular features. The odontogenic potential (capacity to generate teeth) initially resides in the dental epithelium (also referred to as the competent epithelium), which can induce tooth formation in any mesenchyme of neural crest origin [3]. The competent dental epithelium thickens at the location of the future dentition, generating a uniform strip called the dental lamina. Initiation of individual tooth development is marked by the formation of the tooth placodes, the invaginations of the competent epithelium into the underlying mesenchyme which transpire at specific and predetermined loci. The stages that follow placode formation are marked by the proliferation of the epithelial cells and the transition of the epithelium through various shapes ranging from bud- through cap- to bell-like shape, which determines the shape of the future crown.

The placodal stage coincides with the shift of the odontogenic capacity to the underlying neural crest-derived dental mesenchyme which thereafter can induce tooth formation when recombined with epithelium $[3,4]$. In addition, around this time the uniform dental mesen- 
chyme starts to condense. What regulates mesenchymal condensation and what the role of this phenomenon is, which is also observed during the development of other organs, are not fully understood. Mammoto et al. [5] proposed antagonistic interactions between epithelially derived $F g f 8$ and Sema3 $f$ as the inducers of the mesenchymal condensation. The authors further demonstrate that the mesenchymal condensation alone is sufficient to induce the expression of odontogenic markers $\operatorname{Pax} 9$ and Msx1, as well as BMP4, the first epithelial signal associated with the shift of the odontogenic potential to the mesenchyme [2]. The condensed dental mesenchyme initially surrounds the invaginating epithelium, but later becomes encompassed by the epithelium (also referred to as enamel epithelium).

The late bell stage is characterized by cellular differentiation and the production of the mineralized matrices, namely enamel and dentin which form the crown of the tooth. These form at the interface between the inner layer of the dental epithelium (also called inner enamel epithelium) and dental mesenchyme (dental papilla). Cells of the inner dental epithelium differentiate into ameloblasts, while dental papilla cells differentiate into odontoblasts [2]. Generation of both ameloblasts and odontoblasts occurs through stages which culminate in the production of enamel and dentin, respectively. Completion of tooth crown development marks the initiation of root formation. The final steps of root development occur during and after tooth eruption and are characterized by further root elongation and the formation of periodontal tissues which anchor the tooth to the bone.

\section{Age-Related Changes in Human Teeth}

The majority of human organs are composed of cells of limited life span, yet the organs maintain their physical properties and function throughout life. The rejuvenation of the organ cellular and extracellular structure (regeneration) depends on the presence of stem cells, a population of self-renewing cells which can reproduce the cellular heterogeneity of the adult organ over the years. Humans steadily lose their tooth stem cells early in life, and thus their ability to repair detrimental changes that occur throughout life is almost absent.

Teeth are complex structures composed of three mineralized tissues: enamel, dentin, and cementum, which enclose the soft tissues (dental pulp, nerves, and blood vessels). The nonvital enamel is the protective cover of the tooth which is progressively worn, discolored, and less

Biology Explaining Tooth Repair and Regeneration permeable with aging [6]. Injuries or damage to the enamel layer cannot be regenerated due to a loss of ameloblasts at the onset of tooth eruption [6].

Dentin mineralized matrix is produced by odontoblast cells which are generated from the tooth mesenchyme. Unlike enamel, dentin mineral content increases with age due to continuous mineral deposition either as physiological secondary dentin or as tertiary dentin following injury. Gradual changes in the dentin mechanical properties together with weakening enamel increase the potential for tooth fracture.

\section{Tooth Stem Cells}

Studies in animal models have shown that during tooth development, several stem cell niches form in the tooth. The epithelial stem cell niche, also called the cervical loop (Fig. 2), is most morphologically distinct. The cervical loop develops after the bud stage, at the apical end of the advancing epithelium, and it is composed of the inner and outer dental epithelium which forms a loop that encircles the loosely arranged stellate reticulum cells [2]. Stem cells also reside in the stellate reticulum, but the timing of their appearance in the cervical loop is not yet resolved. During the late bell stage, cellular differentiation is initiated at the tips of the future crown cusps by direct contact between the inner enamel epithelium and dental papilla cells. A wave of differentiation of inner enamel epithelium into ameloblasts progressively advances in the apical direction, until it reaches the cervical loop [2]. Further progression of ameloblast differentiation is supported by the stem cells in the cervical loops. Whether this indicates the activation of the stem cell niche is not known. This stem cell niche remains active until the onset of root formation, which coincides with the loss of stellate reticulum from the cervical loops and subsequent loss of epithelial stem cells (Fig. 2) [2, 7]. Subsequently, during tooth eruption, the entire epithelial compartment, including ameloblasts, is lost through cell death mechanisms, including apoptosis [8]. This loss of the epithelial compartment together with the preceding loss of epithelial stem cells and their niche explains the inability of human teeth to regenerate enamel.

\section{Tooth Epithelial Stem Cells}

Tooth epithelial stem cells were first identified in the continuously growing mouse incisors. Smith and Warshawsky [9] in 1975 identified a population of label-retaining cells, which was later shown to be the stem cell 


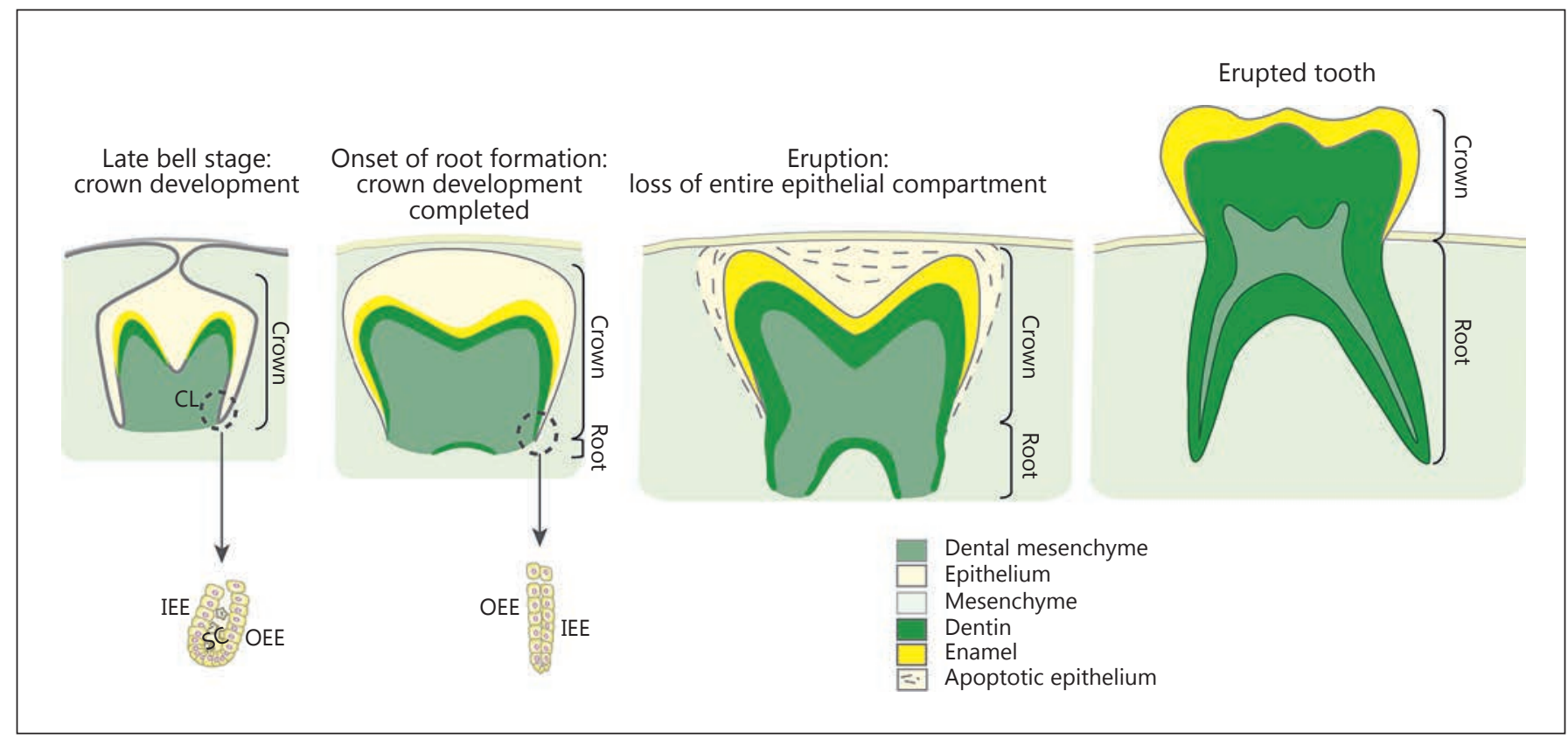

Fig. 2. Late stages of tooth development and eruption. During crown development stem cells (SC) are located in cervical loops composed of inner (IEE) and outer enamel epithelium (OEE) encompassing the stellate reticulum. The disappearance of stellate reticulum and, consequently, the SCs from cervical loop leads to formation of Hertwig's epithelial root sheet (HERS) and the initia-

population capable of generating all epithelial tissues of the tooth $[9,10]$. Since then various genes have been associated with the tooth epithelial stem cell population, including Sox2, Bmi1, Lgr5, ABCG2, Oct3/4, Yap, Gli1, and Lrig1, expressed in overlapping and nonoverlapping patterns [11-16]. How the observed molecular heterogeneity reflects on the differentiation potential and the lineage specificity or exclusivity of the tooth epithelial stem cells is not fully understood. Tooth epithelial stem cells originate from Sox $2+$ cells of the competent dental epithelium, but it is not known whether they retain the inductive odontogenic potential of the epithelium from which they derived [11]. Sox $2+$ tooth epithelial stem cells maintain the competence to generate teeth, which is best observed during the formation of replacement teeth. Human replacement teeth develop from the precisely patterned extensions of the dental lamina which also house Sox $2+$ epithelial stem cells. Lineage-tracing studies in animal models have indicated that the epithelial compartment of the replacement teeth derives from the Sox2+ stem cells residing within the dental lamina extensions, which suggests that Sox2+ cells are competent to participate in tooth formation [17]. tion of root formation. During tooth eruption the entire epithelial compartment, including ameloblasts, is lost through cell death mechanisms. The fully erupted tooth is a complex structure composed of dentin and enamel mineralized tissues enfolding the soft tissues (dental pulp, blood vessels, nerves, etc.).

Collectively, these studies indicate a remarkable plasticity of tooth epithelial stem cells which could potentially be used to generate new teeth de novo. Because the tooth epithelial stem cells are lost in humans, alternative cell sources have to be identified, such as induced pluripotent stem cells (iPSCs; discussed later). However, the fundamental concepts of the regulation of tooth epithelial stem cells are still lacking sufficient information to be successfully utilized toward producing a new cell source which would recapitulate functional and molecular characteristics of tooth epithelial stem cells. So far, studies in animal models have demonstrated that Fgf10 is a major regulator of the tooth epithelial stem cell niche and the disappearance of $\mathrm{Fg} 10$ was implied as the molecular switch necessary for the initiation of root formation [18, 19]. A recent study also showed that increased activity of Shh signaling maintains the stem cell niche in the molar cervical loops. This effect of Shh depended on antagonistic interactions with the BMP signaling pathway, and this signaling cross-talk played a key role in the determination of epithelial stem cell fate [20]. The balance between stem cell proliferation and their differentiation toward the ameloblast lineage is regulated by FAK-YAP-mTOR signal- 
ing [21]. Proper patterning of the enamel derived from stem cells in the cervical loop also depends on the expression of transcription factor Isl 1 and the activity of the Shh signaling pathway $[13,22]$. Modulation of these molecules may result in the generation of ameloblast lineage precursors with characteristics of tooth epithelial stem cells that could be used for regenerative purposes.

\section{Tooth Mesenchymal Stem Cells}

Tooth mesenchymal stem cells are of neural crest origin, located in the postnatal tooth, and are a part of the dentin-pulp complex which has the capacity to repair dentin in response to injury [23]. Based on the cellular origin, two types of dentin can be secreted in response to injury. Mild injury to the dentin which does not reach the odontoblast layer stimulates deposition of the reactionary dentin, which is produced by pre-existing odontoblasts and is structurally similar to physiological dentin. Severe damage, which destroys dentin and the underlying odontoblasts, requires generation of new odontoblast-like cells from the mesenchymal stem cells in the dental pulp. These cells produce reparative dentin which is a poorly organized, mainly atubular structure with cells trapped within the matrix [23]. Mesenchymal stem cells are also involved in the continuous deposition of dentin which ultimately decreases the size of the pulp chamber and consequently the cellularity and vascularity of the dental pulp [6].

Unlike tooth epithelial stem cells, the mesenchymal stem cells do not form a niche that is morphologically distinguishable. Data obtained on continuously growing mouse incisors suggest that there are several niches of mesenchymal stem cells in the tooth, including the perivascular niche (Gli1+ perivascular cells, NG2+ pericytes) and the peripheral nerve-associated, niche-containing cells of glial origin [24-26]. Of these, NG2+ pericytes have been shown to actively participate in injury repair [27]. Studies in human teeth have demonstrated that perivascular cells with stem cell capacity were expressing CD146 and STRO-1 [28]. A recent lineage-tracing study demonstrated that the new odontoblasts generated during reparative dentinogenesis in molar teeth originate from the perivascular cells identified by $a$-smooth muscle actin (aSMA) expression [29]. This study further showed that the progeny of the $\alpha \mathrm{SMA}+$ population participated very little in physiological dentin deposition. Taken together, these studies indicate that the mesenchymal stem cells which actively participate in dentin repair are recruited into the tooth through the vasculature and peripheral nerves.

Biology Explaining Tooth Repair and Regeneration
What stimulates dental mesenchymal stem cells to mobilize to the site of injury and differentiate into a new generation of odontoblast-like cells is not known. Several studies have implicated several cytokines and growth factors, including TGF- $\beta$, but the precise mechanism which governs this process remains obscure [30]. Therefore, further studies are necessary to unravel the inductive signaling, which could then potentially be used for clinical applications in preserving pulp vitality.

The periodontal ligament represents the biggest challenge in reparative and regenerative dentistry, mainly due to its elusive origin and the cellular and molecular properties of this structure in vivo. Recent lineage-tracing studies in the continuously growing incisor demonstrated that the periodontal ligament derived in part from mesenchymal Igfbp5+ and Lrig1+ progenitors, while others have shown that fragmented root epithelial cells also participate in periodontal ligament structure $[16,31]$. Stem cells expressing STRO-1 and CD146 were identified in the periodontal ligament and suggested as a source of regenerative capacity [32].

\section{Tooth Generation de novo}

Epithelial-mesenchymal tissue interactions are the hallmark of tooth development and were first recapitulated in vitro by Kollar and Baird [33]. These authors generated tooth germs in vitro from recombined embryonic dental epithelium and mesenchyme [33]. When grafted into the anterior chamber of the mouse eye, the recombinants grew into properly patterned teeth with mineralized tissues, namely dentin and enamel [4]. In 2007, Nakao et al. [34] generated fully developed teeth by recombining dissociated embryonic tooth germ cells. In the ensuing years, this method has been repeated and further improved $[34,35]$. In these experiments single cell suspensions of separate epithelial and mesenchymal cells were obtained from embryonic tooth germs. Epithelial and mesenchymal cells were recombined within a collagen drop and subsequently recreated the tooth germ which grew to early bell stage in the following 5-7 days of in vitro organ culture. When transplanted into the jaw, at the site where the original tooth resided prior to extraction, these germs developed into fully grown teeth which erupted, occluded, and reconstituted all functions of a normal tooth $[34,35]$.

Generating a tooth de novo in the postnatal animal was a breakthrough in the field, proving that recombination of competent mesenchyme and dental epithelium recapitulates tooth formation, even after disaggregation of 
all tissues involved. However, the Nakao method as such is not applicable in humans, because cells obtained from embryonic tooth germs are not a sustainable or an easily accessible cell source due to practical, ethical, and legal issues. iPSCs, on the other hand, offer a valuable and powerful tool to produce an unlimited cell source for regenerative purposes. The use of iPSCs in organ bioengineering for transplantation purposes has marked a new era in medical research. Various organ rudiments, including the mini-brain and the mini-kidney, have been successfully grown ex vivo from iPSCs $[36,37]$, but so far not the tooth. This is mainly due to a lack of information on how to successfully program the dental identity of iPSCs, which would enable them to reproduce the complex and precisely orchestrated cellular and molecular interactions which occur during in vivo tooth development. Published data demonstrate the production of dental mesenchyme from mouse iPSCs, which can potentially be used as a source of inductive odontogenic potential for tooth regeneration de novo [38]. However, (competent) dental epithelium has not yet been generated from the iPSCs. In addition, dental mesenchymal cells generated from iPSCs, while molecularly similar to the respective dental tissue, have not yet been tested for their ability to participate in and/or induce tooth formation.

\section{Regulation of Tooth Size and Shape}

The most evident drawback in the regeneration of teeth is the inability to generate them of proper shape and size. Tooth shape is determined by epithelial morphogenesis. From the initial stages of tooth development, epithelial morphogenesis is regulated by signaling centers which transiently appear in the dental epithelium during tooth development. These are clusters of tightly packed, nonproliferative cells characterized by the restricted expression of several genes including $p 21, E d a r, D k k 4$, and Fgf20 among others [2]. The first signaling center forms in the placodes and is called the initiation knot. It governs the budding of the dental epithelium and the condensation of the adjacent neural crest-derived mesenchyme. At the tip of the newly formed bud appears the second signaling center called the primary enamel knot which governs tooth crown development. Mammals, including humans, have multicusped teeth in which a third generation of signaling centers (secondary enamel knots) emerges during the bell stage, marking the location of the future cusp. Hence, secondary enamel knots determine the cusp pattern, which is a requisite for proper occlusion.
After the formation of the cusp pattern, the tooth grows to its final size, and mesenchymal odontoblasts and epithelial ameloblasts differentiate at the epithelial-mesenchymal interface to form dentin and enamel, respectively [2].

Signaling centers express several conserved signaling molecules, including FGF, BMP, Wnt, and Shh, which regulate tooth development in a stage- and cell-specific manner [39]. Spatially refined interactions between these signaling pathways determine the cusp number and tooth morphology [39]. It has been previously shown that simultaneous adjustment of Shh, EDA, and activin A pathways increases the complexity of mouse teeth and the number of cusps [40]. Therefore, a system which would enable modulating the activity of various pathways might be necessary for the proper patterning of the secondary enamel knots. Furthermore, this study indicated that an increase in the cusp number does not result from an increase in the tooth size. The tooth crown develops during the late bell stage and continually increases in size until the root formation commences. These data suggest that prolonging the activity of the tooth epithelial stem cell niche may be a strategy to influence tooth size. Therefore, understanding the regulation of stem cells is a crucial tool in order to adequately manipulate the engineered tooth germs toward reaching the correct/appropriate size before the completion of crown development.

Tooth size, on the other hand, is determined by the yet unknown intrinsic signaling which resides in the dental mesenchyme and is thought to be preserved even after cell disaggregation [41]. However, tooth regeneration studies have shown exactly the opposite, since the developed tooth did not have all the morphological and spatial properties of the mouse tooth from which it derived. It has been proposed that the proper tooth size can be achieved by increasing the number of dental mesenchymal cells, which in combination with the accurately shaped bioengineered scaffold could potentially result in the properly sized and shaped tooth. However, the available data demonstrate that changing the number of cells in the dental mesenchyme has no effect on tooth size [41].

\section{Concluding Remarks}

Successful reconstruction of the tooth organ de novo and in vitro depends on our understanding of the cellular and molecular programs which govern its development in vivo. Basic developmental biology studies on various (genetic) animal models are a valuable tool and a prereq- 
uisite for designing novel cell-based strategies for preventing and treating dental defects. In the past decades we have made significant advances in unraveling signaling cascades and cellular events which mediate and/or govern tooth development in vivo. We have identified stem cells which participate in tooth development and postnatal repair, and have uncovered some of the molecular net- works which regulate these populations and that could potentially be used to generate dental stem cells from alternative sources. Most importantly, the tooth regeneration method established by Nakao et al. [34] and further improved by Ikeda et al. [35] provides a great platform on which to build a protocol which will enable the generation of properly sized and shaped teeth.

\section{References}

$>1$ Juuri E, Balic A: The biology underlying ab- $>14$ normalities of tooth number in humans. J Dent Res 2017;96:1248-1256.

-2 Balic A, Thesleff I: Tissue interactions regulating tooth development and renewal. Curr Top Dev Biol 2015;115:157-186.

-3 Mina M, Kollar EJ: The induction of odontogenesis in non-dental mesenchyme combined with early murine mandibular arch epithelium. Arch Oral Biol 1987;32:123-127.

-4 Kollar EJ, Baird GR: Tissue interactions in embryonic mouse tooth germs. II. The inductive role of the dental papilla. J Embryol Exp Morphol 1970;24:173-186.

$\checkmark 5$ Mammoto T, et al: Mechanochemical control of mesenchymal condensation and embryonic tooth organ formation. Dev Cell 2011;21: 758-769.

6 Nanci A: Ten Cate's Oral Histology: Development, Structure, and Function, ed 9. St. Louis, Elsevier, 2018.

$>7$ Thesleff I, Tummers M: Tooth Organogenesis and Regeneration. Cambridge, StemBook 2008.

-8 Kaneko H, Ogiuchi H, Shimono M: Cell death during tooth eruption in the rat: surrounding tissues of the crown. Anat Embryol (Berl) 1997; 195:427-434.

>9 Smith CE, Warshawsky H: Cellular renewal in the enamel organ and the odontoblast layer of the rat incisor as followed by radioautography using ${ }^{3} \mathrm{H}$-thymidine. Anat Rec 1975;183:523561.

10 Harada $\mathrm{H}$, et al: Localization of putative stem cells in dental epithelium and their association with Notch and FGF signaling. J Cell Biol 1999; 147:105-120.

11 Juuri E, et al: Sox2+ stem cells contribute to all epithelial lineages of the tooth via Sfrp5+ progenitors. Dev Cell 2012;23:317-328.

$>12 \mathrm{Li} \mathrm{L}$, et al: Expression patterns of ABCG2, Bmi-1, Oct-3/4, and Yap in the developing mouse incisor. Gene Expr Patterns 2011;11: 163-170.

$\checkmark 13$ Seidel K, et al: Hedgehog signaling regulates the generation of ameloblast progenitors in the continuously growing mouse incisor. Development 2010;137:3753-3761.
Suomalainen M, Thesleff I: Patterns of Wnt pathway activity in the mouse incisor indicate absence of Wnt/ $\beta$-catenin signaling in the epithelial stem cells. Dev Dyn 2010;239:364372.

15 Biehs B, et al: BMI1 represses Ink4a/Arf and Hox genes to regulate stem cells in the rodent incisor. Nat Cell Biol 2013;15:846-852.

16 Seidel K, et al: Resolving stem and progenitor cells in the adult mouse incisor through gene co-expression analysis. Elife 2017;6:e24712.

17 Juuri E, et al: Sox 2 marks epithelial competence to generate teeth in mammals and reptiles. Development 2013;140:1424-1432.

18 Yokohama-Tamaki $\mathrm{T}$, et al: Cessation of Fgf10 signaling, resulting in a defective dental epithelial stem cell compartment, leads to the transition from crown to root formation. De velopment 2006;133:1359-1366.

19 Harada H, et al: FGF10 maintains stem cell compartment in developing mouse incisors. Development 2002;129:1533-1541.

20 Li J, et al: BMP-SHH signaling network controls epithelial stem cell fate via regulation of its niche in the developing tooth. Dev Cell 2015;33:125-135.

$21 \mathrm{Hu} J K$, et al: An FAK-YAP-mTOR signaling axis regulates stem cell-based tissue renewal in mice. Cell Stem Cell 2017;21:91-106.e6.

22 Naveau A, et al: Isl1 controls patterning and mineralization of enamel in the continuously renewing mouse incisor. J Bone Miner Res 2017;32:2219-2231.

23 Sloan AJ, Waddington RJ: Dental pulp stem cells: what, where, how? Int J Paediatr Dent 2009; 19:61-70.

$>24$ Kaukua N, et al: Glial origin of mesenchymal stem cells in a tooth model system. Nature 2014;513:551-554.

25 Zhao H, et al: Secretion of Shh by a neurovascular bundle niche supports mesenchymal stem cell homeostasis in the adult mouse incisor. Cell Stem Cell 2014;14:160-173.

26 Feng J, et al: Dual origin of mesenchymal stem cells contributing to organ growth and repair. Proc Natl Acad Sci USA 2011;108:6503-6508.

27 Pang YW, et al: Perivascular stem cells at the tip of mouse incisors regulate tissue regeneration. J Bone Miner Res 2016;31:514-523.
28 Shi S, Gronthos S: Perivascular niche of postnatal mesenchymal stem cells in human bone marrow and dental pulp. J Bone Miner Res 2003;18:696-704.

29 Vidovic I, et al: aSMA-expressing perivascular cells represent dental pulp progenitors in vivo. J Dent Res 2017;96:323-330.

30 Arany PR, et al: Photoactivation of endogenous latent transforming growth factor- $\beta 1 \mathrm{di}-$ rects dental stem cell differentiation for regeneration. Sci Transl Med 2014;6:238ra69.

-31 Itaya S, et al: Hertwig's epithelial root sheath cells contribute to formation of periodontal ligament through epithelial-mesenchymal transition by TGF- $\beta$. Biomed Res 2017;38:6169.

32 Seo BM, et al: Investigation of multipotent postnatal stem cells from human periodontal ligament. Lancet 2004;364:149-155.

33 Kollar EJ, Baird GR: The influence of the dental papilla on the development of tooth shape in embryonic mouse tooth germs. J Embryol Exp Morphol 1969;21:131-148.

34 Nakao K, et al: The development of a bioengineered organ germ method. Nat Methods 2007;4:227-230.

35 Ikeda E, et al: Fully functional bioengineered tooth replacement as an organ replacement therapy. Proc Natl Acad Sci USA 2009;106: 13475-13480.

36 Takasato M, et al: Kidney organoids from human iPS cells contain multiple lineages and model human nephrogenesis. Nature 2016; 536:238.

37 Lancaster MA, et al: Cerebral organoids model human brain development and microcephaly. Nature 2013;501:373-379.

38 Otsu K, et al: Differentiation of induced pluripotent stem cells into dental mesenchymal cells. Stem Cells Dev 2012;21:1156-1164.

39 Jernvall J, Thesleff I: Tooth shape formation and tooth renewal: evolving with the same signals. Development 2012;139:3487-3497.

40 Harjunmaa E, et al: On the difficulty of increasing dental complexity. Nature 2012;483: 324-327.

41 Cai J, et al: Patterning the size and number of tooth and its cusps. Dev Biol 2007;304:499507 